\title{
CORRECTION
}

\section{Correction to: Comparing head gesture, hand gesture and gamepad interfaces for answering Yes/No questions in virtual environments}

Jingbo Zhao ${ }^{1,2}$ (D) Robert S. Allison ${ }^{2}$

Published online: 9 December 2019

๑) Springer-Verlag London Ltd., part of Springer Nature 2019

\section{Correction to: Virtual Reality} https://doi.org/10.1007/s10055-019-00416-7

In the original publication of the article, the set of Equations 1 was wrongly typeset.

The set of Equations 1 should read as given below

$$
\begin{aligned}
P_{i}^{x} & =\left(F_{i}-C\right) \cdot(n \times h) \\
P_{i}^{y} & =\left(F_{i}-C\right) \cdot h \\
P_{i}^{z} & =\left(F_{i}-C\right) \cdot n
\end{aligned}
$$

The original publication of the article has been updated to reflect the change.
Publisher's Note Springer Nature remains neutral with regard to jurisdictional claims in published maps and institutional affiliations.

The original article can be found online at https://doi.org/10.1007/ s10055-019-00416-7.

\section{Jingbo Zhao}

jingbo@eecs.yorku.ca

Robert S. Allison

allison@eecs.yorku.ca

1 College of Information and Electrical Engineering, China Agricultural University, No. 17 Tsinghua East Road,

Beijing 100083, China

2 Department of Electrical Engineering and Computer Science, York University, 4700 Keele Street, Toronto, ON M3J 1P3,

Canada 